\title{
Éditeurs, correcteurs et autres (1800-1840)
}

\section{Brigitte Louichon}

\section{OpenEdition}

Journals

Édition électronique

URL : http://journals.openedition.org/recherchestravaux/1214

DOI : 10.4000/recherchestravaux.1214

ISSN : 1969-6434

\section{Éditeur}

UGA Éditions/Université Grenoble Alpes

\section{Édition imprimée}

Date de publication : 15 mai 2004

Pagination : 31-42

ISBN : 2-9518254-3-9

ISSN : 0151-1874

\section{Référence électronique}

Brigitte Louichon, «Éditeurs, correcteurs et autres (1800-1840) », Recherches \& Travaux [En ligne], 64 | 2004, mis en ligne le 20 mai 2019, consulté le 08 septembre 2020. URL : http://

journals.openedition.org/recherchestravaux/1214; DOI : https://doi.org/10.4000/recherchestravaux. 1214 
Brigitte LOUICHON

IUFM d'Aquitaine

\section{Éditeurs, correcteurs et autres (1800-1840)}

La figure de l'éditeur est une figure paradoxale fréquente et a priori relativement transparente de l'auteur. La convoquer, c'est évoquer le roman du XVIII' siècle. Pourtant, cette figure et ses avatars sont toujours fréquents durant les premières décennies du XIX $\mathrm{X}^{\mathrm{e}} \mathrm{M}^{\mathrm{me}}$ de Staël, Benjamin Constant, Stendhal, Balzac, Dumas, et d'autres encore dont nous reparlerons, ont, au seuil de leurs romans, convoqué ces figures traditionnelles et légèrement désuètes.

L'on sait que le roman du XVIII' siècle se développe et se construit contre les attaques d'invraisemblance et d'irréalisme dont sont taxés les textes antérieurs. Pour s'affranchir de ces reproches, les romanciers adoptent les modalités narratives de l'histoire, délaissant une narration pseudo-épique en vogue au XVII' siècle. C'est ainsi que les romans-mémoires et les romans épistolaires apocryphes envahissent le XVIII ${ }^{\mathrm{I}}$. Pour renchérir sur la vraisemblance, le texte se double alors d'un dispositif (qui s'actualise le plus fréquemment en préface $^{2}$ ) dont, pour reprendre les termes de Genette, «la fonctionnalité est la fictionnalité ${ }^{3}$ ". Son enjeu essentiel est en effet de présenter le texte donné à lire comme non-fiction, comme texte dont l'auteur (ou les auteurs dans le

I. D'après George May, Le Dilemme du roman, New Haven et Paris, Yale University Press et PUF, 1963, P. 52, environ un cinquième des romans parus entre 1700 et 1750 ressortit au genre du mémoire apocryphe.

2. Le lieu d'inscription des figures de l'éditeur est souvent mouvant et le seuil séparant texte et hors-texte difficile à définir. Les figures fictives sont parfois intégrées au roman luimême, souvent au début (le premier chapitre de La Vie de Marianne), parfois au milieu du roman (Les Souffrances du jeune Werther) ou à la fin (Jacques de George Sand).

3. Gérard Genette, Seuils, Seuil, coll. "Poétique", 1987, p. 256. 
cas d'une correspondance) est le personnage-narrateur. La dénégation auctoriale est au cœur de ce dispositif. Pourtant, je crois que l'on a trop souvent réduit la préface fictionnelle à un simple échange de rôles entre éditeur fictif et auteur réel. Or, par l'intermédiaire de la figure de l'éditeur s'opère d'abord un double mouvement: d'une part, mise en avant d'un auteur présenté comme réel mais qui n'existe pas et, d'autre part, disparition de l'auteur réel. Mais dans le même temps, l'éditeur fictif - l'editeur du mémoire - vient masquer l'existence de l'éditeur réel - l'éditeur du roman- tandis que le personnage de l'éditeur est encore et dans le même temps, une figure du lecteur, puisqu'il est souvent l'interprète du texte donné à lire. De plus, quand l'auteur affirme qu'il n'est pas l'auteur, il affirme aussi qu'il n'est pas le personnage, non simplement narrateur ou épistolier, mais aussi actant. Il n'est ni celui qui a écrit, ni celui qui a vécu les événements donnés à lire. Il y a donc à la fois dénégation auctoriale et dénégation autobiographique. Ces préfaces fictionnelles sont, de fait, un espace où s'effectue un mouvement assez vertigineux par lequel les instances de la communication romanesque, c'est-à-dire les personnages réels qui tournent autour de la fiction textuelle, échangeant en quelque sorte leur place (le personnage devient auteur mais aussi être réel autonome, l'auteur devient éditeur, l'éditeur devient lecteur) font émigrer la fiction du côté des instances. C'est comme si la charge fictionnelle se trouvait ainsi déplacée: délaissant le texte parce qu'ayant envahi le hors-texte.

Cette analyse ne doit pourtant pas négliger le fait que ces dispositifs ont effectivement fonctionné comme créateurs, non seulement de vraisemblance, mais aussi de pseudo-réalité. En effet, la crédulité du lecteur est grande au XVII' siècle et l'on sait, par exemple, que nombre de contemporains de Rousseau ont cru à l'existence de Julie et de Saint-Preux, et qu'il fallut attendre le $\mathrm{XX}^{\mathrm{e}}$ siècle pour que Les Lettres portugaises soit lu comme un roman. Pourtant, l'on voit aussi que ces dispositifs fictionnels, dès la deuxième partie du XVIII siècle, sont sujets à caution. Dans la préface de $L a$ Nouvelle Héloïse, il y a un jeu très subtil qui s'opère entre fiction et nonfiction, personnages réels et personnages fictifs, éditeur et auteur. Fait de glissements et d'ambiguïté, ce jeu rend indécidable le statut du texte au seuil de sa lecture: "Quoique je ne porte ici que le titre d'éditeur, écrit Rousseau, j'ai travaillé moi-même à ce livre, et je ne m'en cache pas. Ai-je fait le tout, et la correspondance entière est-elle une fiction? [...] Que chacun pense comme il lui plaira ${ }^{4}$. Ainsi, l'éditeur ne dit plus " ceci est vrai " mais " ceci est

\footnotetext{
4. Rousseau, CEuvres complètes, Gallimard, coll. "Bibliothèque de la Pléiade ", t. II, I964, p. 5 .
} 
peut-être vrai, peut-être roman "; cette indécision revient de fait à conférer à un texte qui est peut-être de la fiction la même valeur et le même intérêt qu'un texte non-fictif. La même indécision prévaut à la lecture du dispositif qui ouvre Les Liaisons dangereuses, constitué d'un triptyque composé d'une épigraphe reprenant une phrase de la préface de La Nouvelle Héloüse, d'un avertissement de l'éditeur et d'une préface du rédacteur. Le premier texte vient contredire ("ce n'est qu'un roman ") ce que le deuxième texte affirmera ("ce n'est pas un roman").

A la fin du XVIII siècle, le constat est donc le suivant: les romanciers ont à leur disposition un outil romanesque constitué par la mise en texte possible d'une fiction d'auteur, et ce, en général, au seuil de la fiction. Les caractéristiques sur lesquelles je voudrais insister ici sont les suivantes: premièrement, c'est un dispositif complexe, un espace textuel où l'on ne trouve pas une simple figure de l'auteur mais toute une "population particulière de la préface ${ }^{5}$ " et au sein duquel se développent des mouvements multiples entre les différentes instances de la communication romanesque. Deuxièmement, c'est un outil qui a beaucoup servi et dont la fonction a évolué: alors qu'il visait au départ à soustraire le texte à l'univers romanesque, il permet ensuite de conférer au roman, plus ou moins revendiqué comme tel, la même valeur qu'au récit de vie authentique. Le lecteur est moins crédule, le romancier plus sûr de la valeur de la fiction. Troisièmement enfin, on peut affirmer qu'avec la citation opérée par Laclos de la préface de Rousseau, le texte préfaciel fictionnel est entré en littérature.

La question qui va nous occuper maintenant est celle de l'usage que le romancier du début du $\mathrm{XIX}^{c}$ siècle va faire de cet héritage: quelle fiction d'auteur ou quelle figure paradoxale va-t-il projeter sur le devant de sa propre scène romanesque?

Il existe encore quelques éditeurs fictifs, simples passeurs d'un texte produit par le personnage. C'est le cas dans Obermann (1804) qui contient un texte liminaire nommé "observations " présentant la suite tour à tour comme des "mémoires" puis des "lettres". Adolphe paraît sous le titre suivant: "Anecdote trouvée dans les papiers d'un inconnu et publiée par M. Benjamin Constant" et l'éditeur y affirme: "je n'ai pas changé un mot à l'original ${ }^{6}$ ". Pourtant, ces éditeurs, simples passeurs d'un texte, sont plutôt rares et il y a souvent affirmation d'un travail spécifique d'édition. L'éditeur

5. Henri Mitterand, Le Discours du roman, Seuil, 1980, p. 23.

6. Benjamin Constant, Adolphe, Garnier, 1968, p. I5. 
est celui qui va masquer ou changer le nom des personnages. Il est souvent aussi celui qui corrige plus ou moins le texte initial et l'elague parce que comme le dit Balzac - "le cœur est prolixe ${ }^{7}$.

On sait que le début du XIX' siècle a été un âge d'or pour le roman féminin. Mais il est assez invraisemblable pour une femme, eu égard à la réalité du monde du livre, de se présenter comme éditrice. L'auteur adopte alors une autre figure qui est celle de la narratrice ou de la rédactrice d'un récit qui lui a été directement rapporté par le personnage. $\mathrm{M}^{\mathrm{me}}$ Cottin ouvre Claire d'Albe (r799) par une "Préface de l'auteur" où elle affirme avoir rencontré une personne "qui a joué un rôle assez important dans cette histoire. Je lui demandai la permission d'écrire son récit, elle me l'accorda ${ }^{8}$ ". À travers cette figure, l'auteur se fait alors passeur non point d'un texte mais d'une histoire. Ce qui est important, c'est l'authenticité des événements rapportés, la non fiction du personnage plutôt que du texte. $\mathrm{M}^{\mathrm{me}}$ de Krüdener, avec Valérie (1803), reprend le même procédé mais de manière plus élaborée, en copiant celui qui est à l'œuvre dans Werther. Elle rapporte que "l'histoire d'un jeune suédois, d'une naissance illustre, [lui] fut racontée par la personne même qui avait été la cause innocente de son malheur». Elle obtient aussi des textes de ce jeune homme mais ne peut que les parcourir, elle prend des notes, elle change les noms, les lieux, les dates, remplit les lacunes, ajoute des détails. Elle tente "d'imiter la langue simple et passionnée de Gustave 9 ". Ainsi, la fiction de la non-fiction repose ici sur la véracité des faits, l'existence réelle des personnages mais aussi l'existence de textes authentiques dont le texte romanesque ne serait qu'une imitation, une réécriture. On peut paradoxalement affirmer que lorsqu'une romancière convoque la figure de l'éditeur fictif, elle fait preuve d'invraisemblance. Dès lors le dispositif n'a plus d'effet réaliste mais il inscrit au contraire le texte dans le genre romanesque. Sophie Gay, en I802, propose avec Laure d'Estell un roman épistolaire et elle adopte la figure de l'éditeur pour affirmer que les lettres lui ont été transmises par l'héroïne, juste avant qu'elle ne monte sur l'échafaud. Cette préface précède un roman qui, j’ai tenté de le démontrer ailleurs ${ }^{10}$, est un jeu autour du genre sentimental.

7. Balzac, Mémoires de deux jeunes mariées, Gallimard, coll. "Folio ", 1969, p. 326.

8. $\mathrm{M}^{\mathrm{mit}}$ Cottin, Claire d'Albe, in Romans de femmes du XVIF siecle, Laffont, coll. "Bouquins", 1996, p. 691.

9. $\mathrm{M}^{\mathrm{me}}$ de Krüdener, Valérie, in Romans..., ibid., p. 836.

ro. Brigitte Louichon, Ces Voix qui se sont tues. Le Roman feminin de 1794 dे 1830 , Thèse, Bordeaux, 1997, t. I, p. 293-302. 
En fait, ce qui frappe à lire les discours préfaciels fictionnels de cette époque, c'est la disparition des figures pures, simples et des discours univoques. Les dispositifs deviennent plus complexes, souvent doubles. Il y a d'abord - et cela existe déjà depuis La Vie de Marianne avec la figure de l'éditeur doublée de celle du découvreur, dans la Nouvelle Héloïse avec sa double préface ou Les Liaisons dangereuses - les dédoublements. Dans Adolphe, le texte présenté comme authentique est précédé d'une préface de l'éditeur et suivi d'une lettre d'un lecteur du manuscrit qui a connu le personnage "auteur et héros" (lettre elle-même suivie de la réponse de l'éditeur). Ce dispositif, encadrant le texte, encadre aussi le lecteur ou plutôt sa lecture. Le dispositif vise moins à authentifier l'anecdote qu'à lui donner une - ou, en l'occurrence, plusieurs - significations. Autre procédé de dédoublement, la différence que l'éditeur fait entre le texte et le livre. Si l'éditeur de Volupté précise bien que l'auteur est "le personnage non-fictif du récit ${ }^{11}$ ", le récit n'est pas le livre. Le livre est un objet qui transforme le texte initial simplement en le transposant dans un autre contexte communicationnel. L'enjeu pragmatique se déplace et, pour l'éditeur, se pose la question de la pertinence de ce discours qui tout en étant le même devient un autre. L'éditeur se pose alors "longuement» "des questions de conscience» en concluant que "la publication d'un livre vrai aurait peine à être un mal de plus ${ }^{12}$ ». Si le texte devient livre, il doit être doté d'un titre et le choix de "Volupté", avant d'être ou non équivoque (ce dont discute l'éditeur), présente surtout la particularité de se donner comme un titre romanesque. De la même manière, comme l'affirme l'éditeur des Mémoires de deux jeunes mariées (Balzac, 1842), le document authentique doit devenir un "livre, cette chose plus ou moins littéraire qui doit passer sous les yeux du public ${ }^{13}$ ". Pour ce faire, il a une part "dans la correction, dans l'arrangement, dans le choix [des] lettres; mais son travail ne va pas au-delà de celui du metteur en œuvre ${ }^{14}$ ». Quoique Sainte-Beuve et Balzac adoptent pour partie des stratégies différentes (titre romanesque pour le premier, titre centré sur la nature fictive du texte pour le second; travail d'édition différent), ils opèrent une même différenciation entre texte et livre et refusent la posture naïve ou transparente d'un éditeur déconnecté du monde du livre, système au cour duquel il se trouve précisément.

Avec Delphine de $\mathrm{M}^{\mathrm{mc}}$ de Staël (1802), on trouve une autre forme pour le moins équivoque. En effet, sa longue préface est, pour l'essentiel, un plai-

II. Sainte-Beuve, Volupte, Gallimard, coll. “Folio», 1986, p. 31.

12. Ibid., p. 32.

13. Balzac, Mémoires de deux jeunes mariées, op. cit.

14. Ibid., p. 326. 
doyer pour le roman et une histoire du genre. Elle commence d'ailleurs par "Les romans sont de tous les écrits littéraires... ${ }^{15}$ " mais à la fin, l'auteur présente le texte comme "la correspondance que je publie" et affirme "je ne l'aurais pas fait connaître si elle ne m'avait pas paru d'accord avec la manière de voir et de sentir que je viens de développer ${ }^{16}$ ", c'est-à-dire ce que doit être un roman. Il y a là un raccourci assez saisissant, une affirmation que le texte est roman et que l'éditeur est romancier, et pourtant il y a encore recours au vieux procédé, qui est ainsi à la fois exhibé et contesté.

On sent bien, à travers tous ces exemples, que ces dispositifs n'ont plus comme objet de construire une fiction du non-fictif. En 1822, L'Héritière de Birague, l'un des romans de jeunesse de Balzac, s'ouvre sur un «Roman préliminaire c'est-à-dire préface». Ce texte, qui raconte la découverte du cahier qui est ensuite donné à lire, désigne le dispositif comme inopérant. La dimension parodique est le signe de sa perte d'efficacité puisque la préface fictionnelle, pour être opérateur de vraisemblance, doit simuler une préface sérieuse ${ }^{17}$. Dès lors, le roman s'affiche comme tel et la figure de l'éditeur comme le discours liminaire au sein duquel il s'exprime deviennent de fait les marques du genre. En témoignent aussi l'apparition de nouvelles figures. L'avant-propos d'Armance (1827) propose ainsi une nouvelle figure du correcteur, il est celui qui corrige un autre roman. Le texte initial est donc déjà un roman. Il est évident que cette figure-là ne pose plus la même question que le correcteur d'un texte présenté comme non-fictif. De même, la préface du Gars (roman non-écrit mais qui deviendra Les Chouans, 1828) présente la figure de l'éditeur d'un roman d'un auteur fictif nommé Victor Morillon et dont l'éditeur fictif donne une longue biographie.

Il m'apparait donc comme tout à fait évident que ces figures n'ont plus vocation à rendre vraisemblable le roman. Elles opèrent à d'autres niveaux.

On peut par exemple se demander à quelle fin Benjamin Constant adopte cette figure pour Adolphe. En 1817, le procédé est pour le moins galvaudé. D'autant que le romancier, dans la préface fictive, pratique la surenchère: l'auteur nous y emmène en Italie, dans une auberge. L'auteur du manuscrit est un étranger "triste et silencieux", solitaire. Le texte est trouvé par hasard, dans une cassette contenant des lettres, un portrait et un cahier. Bref, Benjamin Constant convoque ici tous les stéréotypes du genre "préface fictionnelle de roman ». L'on peut donc supposer que ce dispositif a pour fonc-

I5. $\mathrm{M}^{\mathrm{me}}$ de Staël, Delphine I, GF-Flammarion, 2000, p. 49.

16. Ibid., p. 58 .

17. Gérard Genette, Seuils, op. cit., p. 257. 
tion première de désigner Adolphe comme roman, c'est-à-dire comme nonautobiographique. Pour Benjamin Constant, se présenter comme éditeur fictif d'Adolphe, revient de fait à endosser le rôle de romancier et donc, dans le même mouvement, à refuser d'endosser le rôle de personnage. On peut supposer que Sainte-Beuve, avec la préface de Volupté, Astolphe de Custine dans Aloys ou les romancières post-révolutionnaires comme Sophie Cottin développent la même stratégie. C'est un peu la même chose qui se passe, quoiqu'à un autre niveau, dans "l'avertissement" de La Chartreuse de Parme. La dénégation n'opère pas au niveau de l'identité du personnage et de l'auteur mais au niveau d'une adhésion possible de l'auteur avec les discours ou les actions de ses personnages. L'auteur en se mettant fictivement en scène affirme: "j'ai rencontré le neveu de la duchesse Sanseverina qui m'a luimême raconté son histoire; j'ai lu les annales rédigées par son mari. En bref, ces personnages ont existé". Mais continue-t-il: "Je déverse le blâme le plus moral sur beaucoup de leurs actions ${ }^{\mathrm{I}}$ " .

Le masque derrière lequel se camoufle l'auteur, s'il le désigne comme romancier, n'en continue pas moins à cacher parfois la personne réelle de l'auteur. La période $1800-1840$ est une période au cours de laquelle le roman conquiert petit à petit ses lettres de noblesse. N'oublions pas que bon nombre des textes cités sont parus anonymement et d'autres sous un pseudonyme (c'est le cas des premiers romans de Balzac). Le roman est encore, dans la hiérarchie littéraire, un genre mineur et souvent féminin mais il est aussi commercialement un produit qui peut se révéler très lucratif ${ }^{19}$. Stendhal écrit à Urbain Canel, l'éditeur d'Armance, qu'il "est tout à fait contre l'usage de mettre son nom à un roman ${ }^{20}$ " même si, comme le lui affirme Mérimée, "vous ne pouvez changer votre style cassant et tous vos lecteurs vous reconnaîtront ${ }^{2 \mathrm{~m}}$. Lorsque Stendhal crée, dans la préface de son premier roman, la figure de correcteur de roman féminin, on peut penser qu'il poursuit trois objectifs: socialement, ne pas se compromettre; littérairement, se donner la possibilité de sortir du roman-mémoire ou épistolaire et adopter la forme de la narration à la troisième personne; et commercialement enfin, espérer le succès d'un roman de femme, à l'instar de ceux de $\mathrm{M}^{\mathrm{me}}$ de Duras avec les-

18. Stendhal, Romans et nouvelles, Gallimard, coll. "Bibliothèque de la Pléiade», r948, t. II, p. 24.

19. Voir Brigitte Louichon, "Editer un roman à succès (1800-1830) ", Eighteenth-Century Fiction, vol. $14, \mathrm{n}^{\circ} 3-4$, avril-juillet 2002 , p. $751-764$.

20. Stendhal, Correspondance générale, Édition de V. del Litto, Honoré Champion, 1999, t. III, p. 644 .

2I. Mérimée, Correspondance générale, Paris, Le Divan, I94I, p. 20. 
quels, on le sait, Armance a beaucoup à voir. De la même manière, lorsqu'il affirme que le roman n'est pas un roman à clés, il joue du stéréotype dénégatif, espérant bien que le lecteur entendra le contraire et que le succès en découlera. Il faut avouer, pour être tout à fait honnête, que ces stratégies furent toutes inefficaces: le roman fut un échec total.

La figure de l'éditeur présente la particularité d'être centrale au sein de l'univers du livre. Il est celui qui en côtoie tous les acteurs, il est donc particulièrement bien placé pour en parler. C'est, nous l'avons vu, cette figure de l'éditeur dont se saisissent Balzac et Sainte-Beuve. Même si l'enjeu en est différent, c'est celle que nous retrouvons dans les "Observations" qui ouvrent Obermann. L'on y trouve la phrase-clé de la préface fictive prototypique: "Ces lettres ne sont pas un roman", l'on y voit un éditeur donnant à lire un texte dont il mesure combien le style est parfois incorrect et les expressions hardies mais qu'il préfere donner dans sa vérité. Ce sont là procédés fort usés. Pourtant, les "observations» d'Obermann ne constituent pas une préface dénégative mais ce que l'on pourrait, pour l'occasion, nommer préface contrastive. L'éditeur - pour justifier son choix - développe en effet tout un système d'oppositions. L'auteur est un "homme qui sent" et non "un homme qui travaille ", le lecteur appartient «à la société éparse et secrète dont la nature avait fait membre celui qui les écrivit" et non des "personnes graves, instruites ou aimables" tandis que le texte est un "monument privé" et non "un roman", "un ouvrage", "un livre raisonnable». Suit toute une série anaphorique: «on y trouvera des passions; mais... on y trouvera de l'amour; mais... on y trouvera des répétitions; mais... etc.":

Je ne me dissimule pas, écrit l'éditeur à la fin du texte, qu'un critique trouvera beaucoup à reprendre: je n'ai point prétendu enrichir le public d'un ouvrage travaillé, mais donner à lire à quelques personnes éparses dans l'Europe, les songes libres et incorrects d'un homme isolé, qui écrivit dans l'intimité, et non pour son libraire ${ }^{22}$.

On voit bien comment l'éditeur fictif construit deux paradigmes : celui de la littérature institutionnelle, et celui d'une autre littérature. Le personnel préfaciel est ici particulièrement important. Du côté de la littérature institutionnelle, aux côtés de l'auteur et du lecteur se trouvent le critique et le libraire. Ces actants fonctionnent en réseau, réseau structuré par certaines notions. C'est de ce côté-ci que se trouvent le travail, l'art, la science, la raison, la dialectique, les systèmes, la froideur. De l'autre, le sentiment, la vérité, l'absence de notoriété, la liberté, la solitude. Mais, par-delà cette opposition

22. Senancour, Obermann, Gallimard, coll. «Folio", 1984, p. 55. 
textes à textes, hantée par le souvenir de Rousseau, se manifeste aussi le désir d'un autre fonctionnement de la sphère littéraire. Lecteurs et auteur du texte idéal se retrouvent dans une même communauté. Les lecteurs sont ici des "adeptes», c'est-à-dire des initiés, les membres d'un groupe partageant les mêmes secrets. L'auteur est "un homme comme eux». De cette sphère idéale, européenne et non plus nationale, communauté de sentiment au lieu de système socio-économique, le critique et le libraire ont bien évidemment disparu. Reste l'éditeur, passeur nécessaire du texte qu'il ne modifie pas, simple agent de constitution de la nouvelle communauté littéraire. Cette figure-là, par-delà celle d'interprète du texte qu'elle donne à lire, tient un discours sur la littérature et le monde au sein duquel elle se produit. C'est en empruntant la figure de l'éditeur comme pivot du monde du livre que l'auteur Senancour appelle de ses voux une nouvelle littérature.

Le désir d'anonymat, l'utilisation parallèle de la figure de l'éditeur et les jeux divers qu'elle autorise sont ici des éléments qui référent au statut du romancier dans un contexte historico-littéraire donné. Écrire un roman oblige l'auteur à se positionner par rapport à la représentation non seulement du roman, mais aussi du romancier construite par l'époque. La lecture de l'avertissement du Gars de Balzac est de ce point de vue assez éclairante. En voici quelques lignes:

Il [l'auteur] manifesta toujours une aversion profonde pour ces préfaces, semblables à des parades où l'on s'efforce de faire croire à l'existence d'abbés, de militaires, de sacristains, de gens morts dans les cachots, et à des trouvailles de manuscrits, qui font épancher sur des créatures postiches tous les trésors de la sympathie. Sir Walter Scott a eu cette manie, mais il a eu le bon esprit de se moquer lui-même de ces superfétations qui ôtent de la vérité à un livre. Si l'on est condamné à monter sur les tréteaux, il faut se résoudre, il est vrai, à y faire le charlatan, mais sans emprunter de mannequin. Nous accueillons avec plus de gravité et d'estime un homme qui se présente modestement en disant son nom et aujourd'hui il y a de la modestie à se nommer, il y a une certaine noblesse à offrir à la Critique et à ses concitoyens une vie réelle, un gage, un homme et non une ombre. [...] S'il a pu exister quelque grâce dans le mystère dont un écrivain s'enveloppe, si le public a respecté son voile comme le linceul d'un mort, tant de barbouilleurs ont usé du rideau qu'à cette heure, il est sali, chiffonné et qu'il n'appartient plus qu'à un homme d'esprit de trouver une ruse nouvelle contre cette prostitution de la pensée qu'on nomme: publication. L'auteur de l'ouvrage que nous publions a donc consenti de bonne grâce à entrer dans la compagnie des illustres danseurs de corde qui, dit-il, s'efforcent, pour de l'argent, d'amuser le public par leurs tours. [...] Je suis pour les tableaux signés, la littérature est une arène où l'on ne veut plus de visières baissées ${ }^{23}$.

23. Balzac, Les Chouans, GF-Flammarion, 1988, p. 413-415. 
Cette préface propose une fiction (Le Gars est écrit par Victor Morillon) mais en cherchant à dépasser le caractère stéréotypé et transparent des procédés de fictionnalisation. En I828, la préface traditionnelle, celle du découvreur de manuscrit, est "parade"; les auteurs fictifs sont des "créatures postiches" et le voile de l'anonymat est devenu un «rideau usé et barbouillé». Et si ces procédés ont perdu de leur efficacité, c'est que le monde et la littérature ont changé. Écrire, c'est "monter sur les tréteaux"; devenir auteur, c'est devenir un de ces "illustres danseurs de corde" et la "littérature est une arène" où les romanciers combattent pour de l'argent. Alors il faut signer ses textes " comme ces bourgeois de Paris qui, sortant leur chien favori, lui mettent un petit collier sur lequel un graveur inscrivit le nom du maître". Ou alors, en "homme d'esprit", il faut «trouver une ruse nouvelle». Et c'est bien sûr ce que fait Balzac, de manière retorse et signifiante. Retorse puisque la dénégation (je ne suis pas l'auteur de ce roman) s'appuie ici sur la dénégation de la dénégation (ceci n'est pas une préface dénégative). Mais aussi signifiante dans la mesure où cette préface contient sa propre clé de lecture. C'est bien sûr la référence à Walter Scott qui a tout à la fois "la manie " des préfaces et le bon goût de s'en moquer. L'on sait en effet que l'auteur écossais a développé tout un jeu préfaciel, un système de personnages d'auteurs et d'éditeurs qui viennent masquer celui que ses contemporains admiratifs nommèrent «le grand inconnu". Mais la référence est ici double. La préface n'est pas un texte autonome, elle fonctionne en dyptique avec le roman qu'elle précède. Balzac écrit une préface à la manière de Scott parce que le roman du Gars est - ou plutôt devait être - un roman à la manière de Scott.

Et ceci nous amène à notre dernier point concernant les enjeux liés aux figures fictives. Lorsque le romancier endosse l'une de ces figures, figure traditionnelle ou figure nouvelle, il cherche à indiquer de quelle manière ce texte s'inscrit dans le contexte historico-littéraire de son époque mais il s'inscrit aussi de fait dans une histoire du genre. L'auteur adopte ou modifie ou crée ce que l'on peut nommer une posture générique. Lorsque Balzac écrit la première préface des Chouans, un an après (I829) celle du Gars, le changement est radical: il signe son roman et dans la préface, c'est le romancier qui s'exprime et qui déclare: "les choses parlent d'elles-mêmes "24". Nous basculons dans une nouvelle ère, qui est celle, pour reprendre les mots de Benveniste qui ressemblent tant à ceux de Balzac, où les romans deviennent des "récits où les événements semblent se raconter eux-mêmes ${ }^{25}$ ". Lorsque

24. Ibid., p. 429.

25. Émile Benveniste, "Les relations de temps dans le verbe français", Problèmes de linguistique générale, Gallimard, 1969, p. $24 \mathrm{I}$. 
Balzac écrit la préface de la Comédie humaine en I842, il y affirme haut et fort les droits et devoirs du roman et dans le même temps décide de republier ses anciens textes sans leurs préfaces originelles. C'est ainsi que Les Mémoires de deux jeunes mariées se voit amputé de sa préface fictive. La figure de l'éditeur de correspondance a disparu et le texte, intégré au corpus balzacien, devient ouvertement et glorieusement un roman, revendiqué commẹ tel. La fiction de la non-fiction devient inutile eu égard à l'enjeu réaliste. On se souvient de la phrase de Flaubert, dans la première Éducation sentimentale, à propos "des balivernes sentimentales que s'écrivent les amants»: «La passion ne se peint pas plus elle-même qu'un visage ne fait son portrait ni qu'un cheval n'apprend l'équitation ${ }^{26}$ ". La fiction est reconnue comme mode d'appréhension du réel en tant qu'elle est fiction. Et pour ce faire, «l'auteur dans son œuvre [ne] doit être visible [...] nulle part ${ }^{27}$ » affirme Flaubert. Plus de figures, plus de masques, plus de préfaces, l'auteur doit disparaître au seul profit de son texte. Ce qui n'est pas sans poser quelques problèmes, dont le procès infligé à Flaubert à propos de Madame Bovary semble un bon exemple. Laisser le texte nu, c'est accepter de n'opérer, au seuil de celui-ci, aucun guidage du lecteur. Or la figure de l'éditeur fictif, peut-être encore plus que celle de l'auteur préfacier de son propre texte, parce qu'elle est discours du premier lecteur (fictif) du texte, autorise un discours contraignant sur le mode de lecture à opérer et le sens à construire.

De sorte que l'on peut peut-être affirmer que le paradoxe le plus criant est celui-ci : lorsqu'un auteur adopte la figure de l'éditeur, c'est-à-dire une figure de non-auteur, il n'en manifeste pas moins une très forte volonté de maîtrise auctoriale sur son texte, sur son lecteur et sur leur rencontre.

La figure fictive de l'auteur, quelle que soit la configuration qu'elle prenne (éditeur, rédacteur, correcteur ou autre), est, je le crois, un exemple de ces objets littéraires qui permettent de comprendre la littérature comme un continuum de discours. En effet, on se rend compte que l'on peut en faire une histoire qui, pour n'être pas totalement linéaire, peut néanmoins être schématisée en grandes phases. Il y a d'abord une phase d'élaboration de la figure qui se situe en gros au XVII et durant la première moitié du XVIII. Puis une phase de déconstruction, de jeu avec elle, qui, je viens de tenter de le

26. Flaubert, Euvres de jeunesse, Gallimard, coll. "Bibliothèque de la Pléiade", 200I, p. 882 .

27. Flaubert, Correspondance II, Gallimard, coll. "Bibliothèque de la Pléiaden, I980, p. 204. 
montrer, se développe entre 1760 et 1840 . Vient ensuite la période de disparition de la figure qui correspond à l'époque réaliste et naturaliste. Il paraît dès lors évident que la phase suivante ne peut être qu'une phase de réapparition, de résurrection. On en trouve un exemple assez jubilatoire dans Le Nom de la rose d'Umberto Eco. Son roman s'ouvre sur un texte intitulé "un manuscrit, naturellement" qui rapporte la découverte du texte. Or, il n'y a en la matière, bien évidemment, rien de "naturel" mais tout au contraire uniquement du culturel. C'est là, comme le souligne le découvreur fictif du manuscrit à la fin de son texte, "une histoire de livres ${ }^{28}$ ", c'est-à-dire un jeu avec les textes, avec la mémoire et le savoir du lecteur, une manière pour chaque texte de s'inscrire dans la grande bibliothèque de l'histoire et du monde.

28. Umberto Eco, Le Nom de la rose, Librairie générale française, coll. "Le livre de poche ", 1982, p. I4. 